\title{
The BH3-only proteins BIM and PUMA are not critical for the reticulocyte apoptosis caused by loss of the pro-survival protein BCL-XL
}

\author{
Alex RD Delbridge ${ }^{\star, 1,2,4}$, Brandon J Aubrey ${ }^{1,2}$, Craig Hyland ${ }^{1}$, Jonathan P Bernardini ${ }^{1,2}$, Ladina Di Rago ${ }^{1}$, Jean-Marc Garnier ${ }^{1}$, \\ Guillaume Lessene $^{1,2,3}$, Andreas Strasser ${ }^{1,2,5}$, Warren S Alexander ${ }^{1,2,5}$ and Stephanie Grabow ${ }^{\star, 1,2,5,6}$
}

Anaemia is a major global health problem arising from diverse causes and for which improved therapeutic strategies are needed. Erythroid cells can undergo apoptotic cell death and loss of pro-survival BCL-XL is known to trigger apoptosis during late-stage erythroid development. However, the mechanism by which loss or pharmacological blockade of BCL-XL leads to erythroid cell apoptosis remains unclear. Here we sought to identify the precise stage of erythropoiesis that depends on BCL-XL. We also tested whether deficiency of BIM or PUMA, the two main pro-apoptotic antagonists of BCL-XL, could prevent reticulocyte death and anaemia caused by BCL-XL loss. Using an in vivo mouse model of tamoxifen-inducible Bc/x gene deletion and in vitro assays with a BCL-XL-selective inhibitor, we interrogated each stage of erythrocyte differentiation for BCL-XL dependency. This revealed that reticulocytes, but not orthochromatic erythroblasts, require BCL-XL for their survival. Surprisingly, concurrent loss of BIM or PUMA had no significant impact on the development of anemia following acute BCL-XL deletion in vivo. However, analysis of mixed bone marrow chimaeric mice revealed that loss of PUMA, but not loss of BIM, partially alleviated impaired erythropoiesis caused by BCL-XL deficiency. Insight into how the network of pro-survival and pro-apoptotic proteins works will assist the development of strategies to mitigate the effects of abnormal cell death during erythropoiesis and prevent anaemia in patients treated with BCL-XL-specific BH3-mimetic drugs.

Cell Death and Disease (2017) 8, e2914; doi:10.1038/cddis.2017.304; published online 6 July 2017

Anaemia affects over 1.5 billion people world-wide ${ }^{1}$ and is a major cause of morbidity that requires improved therapeutic interventions. Underlying causes for anaemia are diverse and include haemoglobinopathies, red cell enzyme disorders, nutritional deficiencies, cytoskeletal abnormalities and autoimmune diseases. Furthermore, anaemia is a frequent toxicity associated with cancer therapy, including both conventional chemotherapy as well as emerging targeted therapies, such as some of the $\mathrm{BH} 3-$ mimetics. ${ }^{2,3}$ Understanding the requirements for normal erythroid development and survival will help develop improved treatment strategies to support erythropoietic function in patients.

In the adult, mature red blood cells derive from haematopoietic stem cells. Erythropoiesis proceeds through several differentiation steps whereby the potential for alternate blood cell lineages is progressively lost and immature progenitors expand in number to facilitate production of sufficient mature red blood cells, with half-lives spanning 100-120 days in humans and 820 days in mice. ${ }^{4-7}$ Should bone marrow erythropoiesis become compromised - due to bone marrow failure/dysplasia or infiltration, or excessive loss/destruction of peripheral red blood cells - extramedullary erythropoiesis can occur in the spleen to facilitate enhanced erythrocyte production in mice. Robust prosurvival signalling is essential to prevent excessive apoptotic cell death during erythropoiesis and the maintenance of adequate red blood cell numbers. Expression analysis has identified the pro-survival gene $B c l x$ as particularly highly expressed in the erythroid lineage, ${ }^{8}$ suggestive of its critical importance for countering apoptosis during erythropoiesis. Aberrant apoptosis of red blood cells and erythroid progenitors is a common feature of chronic anaemias and is likely to contribute to their severity. For example, thalassaemia patients have elevated numbers of early precursor cells in the bone marrow but abnormally high rates of apoptosis in more mature progenitors, ${ }^{9}$ whereas in anaemia of chronic disease, elevated interferon $-\gamma$ levels are associated with increased apoptosis of erythroid progenitors and inversely correlated with reticulocyte numbers and haemoglobin levels. ${ }^{10}$ Aberrant apoptosis is also a key feature of Diamond-Blackfan Anaemia where ribosomal stress drives abnormal TP53 activation and cell death. ${ }^{11}$ TP53 is known to regulate the expression of several key initiators of apoptosis, including Puma, Noxa and Bax. ${ }^{12-15}$

\footnotetext{
${ }^{1}$ The Walter and Eliza Hall Institute of Medical Research, Melbourne, VIC, Australia; ${ }^{2}$ Department of Medical Biology, University of Melbourne, Melbourne, VIC, Australia and ${ }^{3}$ Department of Pharmacology and Therapeutics, University of Melbourne, Melbourne, VIC, Australia

*Corresponding author: ARD Delbridge, Neuroimmunology Research, Biogen, 115 Broadway, Cambridge, MA 02142, USA. Tel: +1 617 914 4670;

E-mail: alex.delbridge@biogen.com

or S Grabow, Department of Cancer Immunology and Virology, Dana Farber Cancer Institute, 450 Brookline Ave, Boston, MA 02115, USA. Tel: +1 617 990 2196;

E-mail: Stephanie_Grabow@dfci.harvard.edu

${ }^{4}$ Current address: Neuroimmunology Research, Biogen, Cambridge, MA, USA

${ }^{5}$ These authors share senior authorship.

${ }^{6}$ Current address: Dana Farber Cancer Institute, Department of Cancer Immunology and Virology, Boston, MA, USA.

Received 18.4.17; accepted 02.5.17; Edited by G Raschellà
} 
The intrinsic (also called 'BCL-2-regulated', 'stress' or 'mitochondrial') apoptotic pathway is regulated by three major classes of proteins. ${ }^{16,17}$ The pro-survival proteins (e.g., BCL-2, BCL-XL, MCL-1) antagonise the pro-apoptotic effectors BAX and BAK. Upon stress stimuli pro-apoptotic BH3-only proteins, such as BIM and PUMA, are induced transcriptionally or post-transcriptionally in a cell type and stimulus-specific manner, and initiate apoptosis by displacing BAX/BAK from their restraint by the pro-survival proteins and possibly also by direct activation of BAX/BAK. ${ }^{18}$ Activated BAX and BAK mediate disruption of the outer mitochondrial membrane. This represents the 'point of no return' for the cell, triggering activation of the caspase cascade, resulting in proteolysis of many substrates and cellular demolition. ${ }^{19}$

In accordance with their roles in apoptosis signalling, loss of pro-apoptotic BIM or combined loss of BAX and BAK result in abnormal accumulation of diverse haematopoietic cell types, $^{20,21}$ whereas conversely loss of pro-survival MCL-1 results in bone marrow failure. ${ }^{22,23}$ In the erythroid lineage $\mathrm{BCL}-\mathrm{XL}$ is of critical importance. Germline deletion of $\mathrm{BClX}$ results in embryonic death of mice at $\sim$ E13.5 in part owing to increased apoptosis of erythroid progenitors; interestingly, this death of erythroid progenitors could be rescued by concomitant
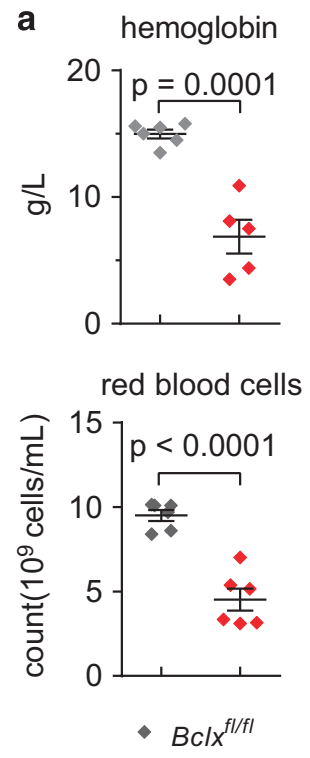

b

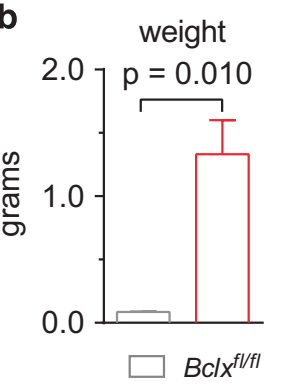

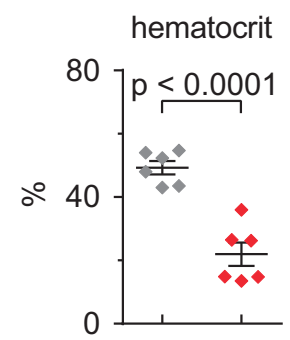

reticulocytes
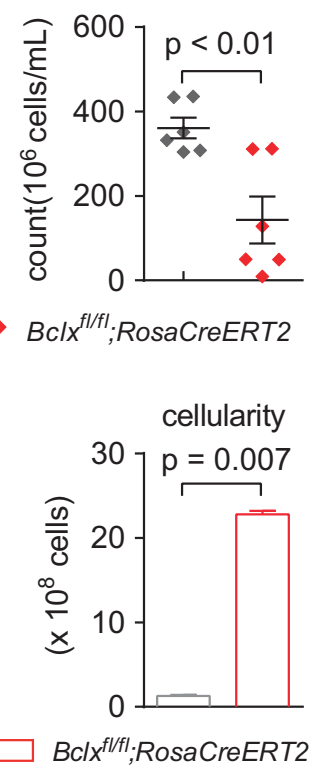

Figure 1 Acute loss of BCL-XL causes severe anaemia. $B c x^{f / f / f} ;$ RosaCreERT2 $2^{K /+}$ mice and control $B c l x^{f / f t}$ mice were treated with tamoxifen (TAM) to induce $B c l x$ gene deletion. After 1 month (a) blood analysis was performed and (b) spleen weight and cellularity were determined. $n=5-6$. Data are presented as mean \pm S.E.M. Significant $P$-values are shown, unpaired Students $t$-test loss of BIM. ${ }^{24,25}$ Tissue-restricted deletion of $B c / x$ results in severe anaemia, splenomegaly due to erythroblast accumulation, and thrombocytopenia. ${ }^{26}$ Loss or inhibition of BCL-XL (e.g., using the BH3-mimetics ABT-737 or ABT-263 ref. 27,28), but not of BCL-2 (e.g., using ABT-199 ref. 29), results in thrombocytopenia in patients and mouse models and also anaemia in mice. ${ }^{2,24,26,27,30-34}$ It has yet to be established whether pharmacological inhibition of BCL-XL will also cause anaemia in patients. Published data demonstrate that both $B A X$ and BAK must be removed to prevent anaemia caused by loss or drug mediated inhibition of BCL-XL, as they have largely overlapping roles in the execution of apoptosis. ${ }^{26,35}$ It is, however, still unknown which $\mathrm{BH}$-only protein is responsible for the initiation of apoptosis in this context.

We defined the requirement for $B C L-X L$ at various stages of adult erythropoiesis by using a discriminating flow cytometry method $^{36}$ and a tamoxifen-inducible, acute $B c l x$ gene deletion mouse model. Given that BIM is essential for the aberrant apoptosis of erythroid progenitors in embryonic mice caused by the absence of BCL-XL, ${ }^{25}$ we investigated the role of this pro-apoptotic $\mathrm{BH} 3-o n l y$ protein in adult erythropoiesis. We found that $B C L-X L$ is critical for the survival of reticulocytes and that BIM is not essential for the anaemia that is caused by acute loss of BCL-XL, whereas pro-apoptotic PUMA has a minor role. These discoveries inform the development of strategies to alleviate anaemia caused, for example, by inherited mutations, infections or treatment with anti-cancer agents, including the new $\mathrm{BH} 3-$ mimetic drugs.

\section{Results}

Acute loss of BCL-XL causes profound anaemia in adult mice owing to failure of erythropoiesis. To confirm and extend published data characterising the role of $B C L-X L$ in erythroid cell survival, ${ }^{24,26,30}$ we generated mice bearing floxed Bclx (Bc/2/1) alleles that could be deleted in an inducible manner by tamoxifen-dependent CreERT2-recombinase activity. $B c l x^{f l}$ mice ${ }^{26}$ were crossed with Rosa$\mathrm{CreERT2}^{\mathrm{Ki}}$ mice $^{37}$ to generate $\mathrm{Bcl} \mathrm{x}^{\mathrm{fl} / \mathrm{fl}} ;$ RosaCreERT2 $^{\mathrm{Ki} /+}$ compound mutant mice. In these mice, tamoxifen administration activates the latent CreERT2 recombinase to facilitate recombination of the floxed $B c / x$ alleles, leading to loss of BCL-XL expression.

A cohort of $B c / x^{f l / f l} ;$ RosaCreERT2 ${ }^{K i /+}$ mice and $B c / x^{f / f l}$ control mice were treated with tamoxifen at 8 weeks of age. At 1 month post treatment these adult mice were analysed to determine the effects of BCL-XL loss. Peripheral blood analysis confirmed previous reports, ${ }^{26,31}$ with profound anaemia observed in the $\mathrm{Bcl} x^{f / f l} ;$ RosaCreERT2 ${ }^{K i /+}$ mice. Haemoglobin $(P=0.0001)$ levels, haematocrit $(P<0.0001)$ and reticulocyte as well as mature red blood cell numbers were all significantly reduced (Figure 1a). Conversely, both spleen weight and cellularity were increased in the $B c / x^{f l / f l} ;$ RosaCreERT2 $2^{K i /+}$ mice (Figure 1b). In contrast, the tamoxifentreated $B c / x^{f l / f l}$ control mice retained normal blood and spleen cell counts.

A previous study reported that haemolysis was associated with $\mathrm{BCL}-\mathrm{XL}$ deletion. ${ }^{26}$ We therefore examined the impact of inducible adult loss of BCL-XL on red blood cell turnover. 


\begin{tabular}{|c|c|c|c|c|c|c|c|}
\hline \multirow[b]{2}{*}{ Bone marrow } & \multicolumn{7}{|c|}{ Number of colonies per 25000 cells } \\
\hline & Total & Blast & G & GM & M & Eo & Meg \\
\hline \multirow[t]{2}{*}{$\begin{array}{l}B c \mid x^{f|/ f| l \mid}(n=6) \\
B c l x^{f / f t} ; C \operatorname{CreRERT2}(n=4)\end{array}$} & $\begin{array}{l}106 \pm 29 \\
114 \pm 21\end{array}$ & $\begin{array}{l}14 \pm 5 \\
21 \pm 4\end{array}$ & $\begin{array}{l}24 \pm 9 \\
26 \pm 7\end{array}$ & $\begin{array}{l}23 \pm 4 \\
24 \pm 8\end{array}$ & $\begin{array}{l}12 \pm 4 \\
14 \pm 4\end{array}$ & $\begin{array}{l}7 \pm 2 \\
7 \pm 4\end{array}$ & $\begin{array}{l}21 \pm 6 \\
23 \pm 5\end{array}$ \\
\hline & \multicolumn{7}{|c|}{ Number of colonies per 50000 cells } \\
\hline Spleen & Total & Blast & G & GM & $\mathbf{M}$ & Eo & Meg \\
\hline $\begin{array}{l}B c \mid x^{f \mid / f l}(n=6) \\
B c l x^{f / f t l} ; C r e R E R T 2(n=4)\end{array}$ & $\begin{array}{c}9 \pm 1 \\
21 \pm 12\end{array}$ & $\begin{array}{l}1 \pm 1 \\
5 \pm 3\end{array}$ & $\begin{array}{l}1 \pm 1 \\
3 \pm 3\end{array}$ & $\begin{array}{l}1 \pm 1 \\
2 \pm 2\end{array}$ & $\begin{array}{l}1 \pm 1 \\
4 \pm 2\end{array}$ & $\begin{array}{l}0 \\
1 \pm 1\end{array}$ & $\begin{array}{l}6 \pm 1 \\
7 \pm 3\end{array}$ \\
\hline
\end{tabular}

Bone marrow cells were cultured in semi-solid agar in the presence of SCF+IL-3+EPO for 7 days. The numbers and types of colonies were scored from dried, stained cultures. Means \pm S.D.s are shown.

Consistent with abnormal red blood cell destruction, we observed an increase in serum levels of unconjugated bilirubin in the tamoxifen-treated $B c / x^{f / / f l} ;$ RosaCreERT2 ${ }^{K i /+}$ mice (Supplementary Figure 1A). Consistent with normal hepatic function, no increases in the levels of serum albumin, alkaline phosphatase, aspartate aminotransferase and gammaglutamyl transferase ( $<4 \mathrm{U} /$; ; data not shown) were observed. There was a marginal increase in alanine aminotransferase; however, the levels observed were below those indicative of significant liver damage (Supplementary Figure 1B). Hence, we conclude that liver dysfunction did not account for the accumulation of serum bilirubin. The serum haptoglobin levels were very low $(<0.08 \mathrm{~g} / \mathrm{l}$; data not shown), consistent with abnormal red blood cell breakdown and release of haemoglobin. However, the lactate dehydrogenase levels were not increased, which suggests that red blood cell destruction may occur predominantly within the extra-vascular space (bone marrow and spleen) (Supplementary Figure 1B).

Blood films were prepared from tamoxifen-treated $\mathrm{Bcl} \mathrm{x}^{\mathrm{fl} / \mathrm{fl} \text {; }}$ RosaCreERT2 $2^{\mathrm{Ki} /+}$ and RosaCreERT2 ${ }^{\mathrm{Ki} /+}$ (control) mice to identify changes in red blood cells caused by loss of BCL$\mathrm{XL}$. Remarkably, almost complete disappearance of reticulocytes was seen by day 2 (Supplementary Figure 2A). This was preceded by morphologic changes in the reticulocytes of coarse basophilic stippling (Supplementary Figure 2B). There was no evidence of spherocytosis, red blood cell fragmentation (schistocytes) or specific changes typical of oxidative haemolysis ('bite' or 'blister' cells) following BCL-XL loss. Mild CRE recombinase toxicity was observed in control animals at day 5 , with a minor reduction in reticulocytes. However, this effect was transient and haematopoietic reconstitution experiments revealed that transient CreERT2 activation exerted no long-term consequences (Supplementary Figure 2C).

Red blood cells constitute $>95 \%$ of all blood cells and must be continuously replaced as they are lost or expended. Consequently, perturbation of bone marrow stem/progenitor cell activity may lead to the observed anaemia. Hence, we examined whether the reduction in red blood cells in the tamoxifen-treated $B c / x^{f / / f l} ;$ RosaCreERT2 $2^{K i /+}$ mice was a consequence of defective bone marrow stem/progenitor cell activity. Flow cytometry was used to determine the proportions and numbers of stem and progenitor cell subsets in the bone marrow of these mice. This revealed that after tamoxifen treatment $B c / x^{f l / f l} ;$ RosaCreERT2 $2^{K i /+}$ and $B c / x^{f l / f l}$ (control) mice had similar proportions and numbers of stem cells (LT-HSC, ST-HSC), multipotent progenitors (MPP) and committed progenitor cells (MEP, CMP, GMP) (Supplementary Figure $3 \mathrm{~A}$ ). As a functional readout of progenitor cells, we performed colony-formation assays with bone marrow and spleen cells in semi-solid agar. No significant differences were observed in the relative proportions of the various myeloidcommitted colony-forming cells in the bone marrow and spleen between tamoxifen-treated $B c / x^{f l / f l} ;$ RosaCreERT2 $2^{K i /+}$ and $B c / x^{f l / f l}$ (control) mice (Table 1). Therefore, we conclude that $B C L-X L$ is dispensable for the survival of haematopoietic stem/progenitor cells in adult mice at steady state.

We next wanted to define the precise stage at which red blood cell production is perturbed to identify the cause of the anaemia caused by loss of BCL-XL. One month after deletion of $B c / x$, red blood cell maturation was analysed by flow cytometry based on expression of TER119, CD44 and cell size (FSC) (ref. 36; Figure 2a). Following Bclx gene deletion we observed significantly decreased percentages of mature red blood cells, but not reticulocytes in the bone marrow with a corresponding increase in the percentages of the more immature precursors (Figure $2 \mathrm{~b}$ ). This phenomenon was mirrored in the spleen of the tamoxifen-treated $B c / x^{f l / f t}$; RosaCreERT2 $2^{\mathrm{Ki}+}$ mice, where normally only few immature erythroid progenitor cells are found. Colony-formation assays revealed a pronounced increase in the numbers of colonyforming units-erythroid (CFU-e) in the spleen of tamoxifentreated $B c / x^{f l / f l} ;$ RosaCreERT2 $2^{K i /+}$ mice compared with $B c / x^{f l / f l}$ mice, whereas no differences were observed in the bone marrow (Supplementary Figure $3 \mathrm{~B}$ ). These findings are consistent with erythropoietic insufficiency in the tamoxifentreated $B c / x^{f l / f l} ;$ RosaCreERT2 $2^{K i / 4}$ mice, whereupon the spleen has been recruited as a site for extramedullary erythropoiesis in a reactive attempt to overcome the low numbers of mature red blood cells in the circulation.

Next, we harvested bone marrow cells from $B c / x^{f l / f l}$; RosaCreERT2 $2^{K i /+}$ and $B c l x^{f l f l}$ mice one month following tamoxifen treatment and tested their ability to reconstitute the haematopoietic system of lethally irradiated recipient mice. The animals reconstituted with $B c / x^{f l / f l} ;$ RosaCreERT2 ${ }^{K i /+}$ cells developed symptoms of erythropoietic failure within 3 weeks post-reconstitution. Blood transfusion was performed, however by 4 weeks post-reconstitution these mice reached ethical endpoint and were killed for analysis. Flow cytometric 
a

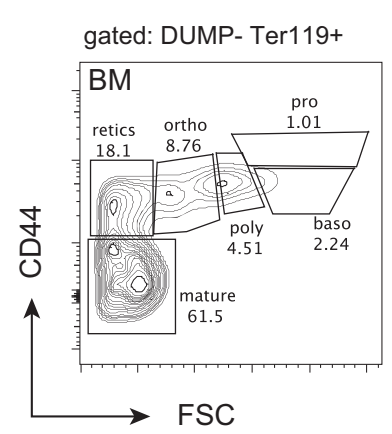

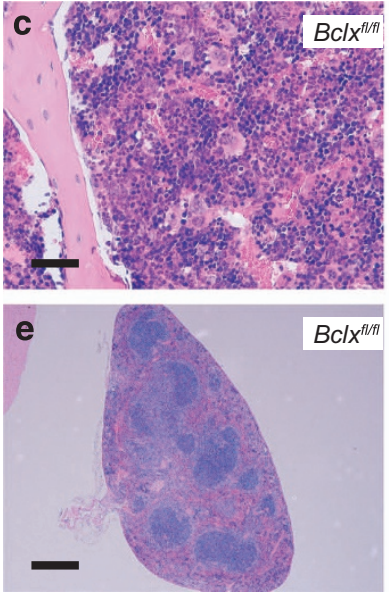

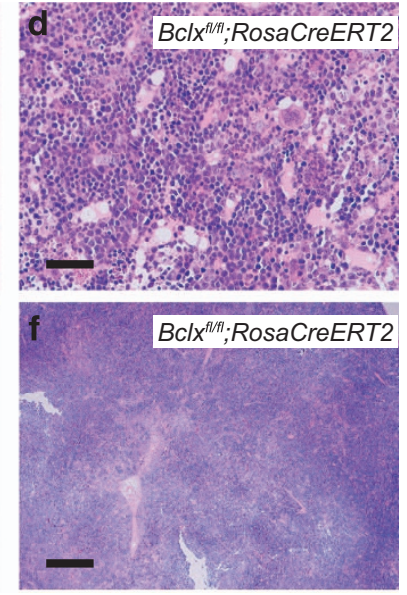

b

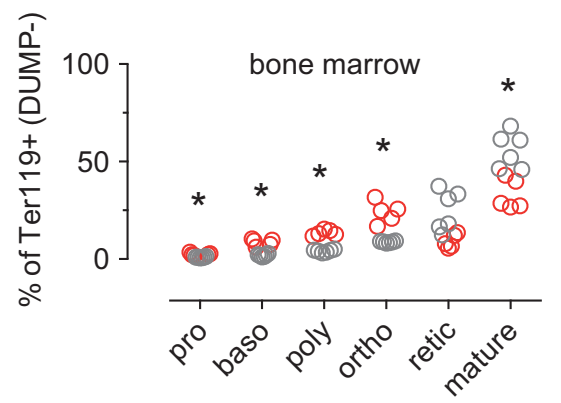

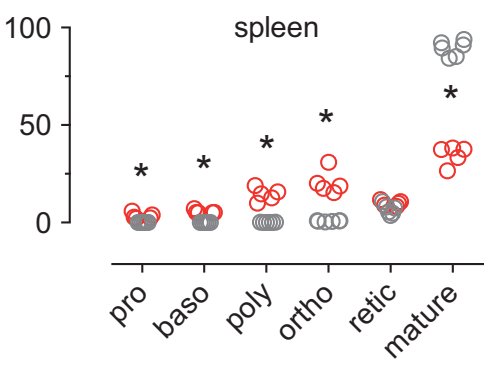

Figure 2 Acute deletion of BCL-XL results in an elevation of erythroid progenitors. $B c l x^{f / f t /} ; R o s a C r e E R T 2^{K / /+}$ mice and control $B c / x^{f / / f t}$ mice were treated with tamoxifen (TAM) to induce $B c l x$ gene deletion. Analysis of bone marrow and spleen was performed after 1 month. (a) Gating strategy using CD44 versus forward light scatter (FSC) to delineate defined erythropoietic stages; in order of increasing maturity: pro-erythroblasts (pro), basophilic erythroblasts (baso), polychromatic erythroblasts (poly), orthochromatic erythroblasts(ortho), reticulocytes (retic) and mature red blood cells (mature). (b) Erythroid subset analysis (\%) in the bone marrow and spleen (Bcl $x^{t / f t} ;$ RosaCreERT2 ${ }^{K K / t}$ in red,

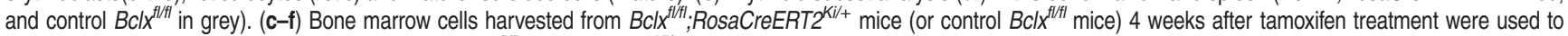
reconstitute lethally irradiated GFP' recipient mice. Bcl $x^{f / f / f} ;$ RosaCreERT2 ${ }^{K / /+}$ bone marrow reconstituted mice developed anaemia after 3 weeks and were transfused with $250 \mu l$ $\mathrm{GFP}^{+}$blood. Histological analysis of sternum $(\mathbf{c}, \mathbf{d})$ and spleen $(\mathbf{e}, \mathbf{f})$ specimens collected from $B c l x^{f / f t /} ;$ RosaCreERT ${ }^{K / /+}$ bone marrow reconstituted mice or control $B c l x^{f / f / t}$ bone marrow reconstituted mice. Bone marrow sections ( $\times 20$ objective) scale bar $=4 \mu \mathrm{m}$, spleen sections ( $\times 40$ objective) scale bar $=20 \mu \mathrm{m}$. Data are representative of $n=3$ mice per genotype. (b) $n=5-6$, unpaired Students $t$-test, adjusted for multiple testing (Holm-Sidak); ${ }^{*} P<0.002$

analysis gated on donor, non-transfused cells (GFP-) in the spleen and bone marrow revealed elevated proportions of immature erythroid progenitors with a decrease in the proportions of reticulocytes and mature red blood cells in the tamoxifen-treated $B c l x^{t / f t} ;$ RosaCreERT2 ${ }^{K i /+}$ mice (Supplementary Figure 4). Although no major architectural changes were observed in the bone marrow of these mice (Figures 2c and d), widespread disruption of splenic architecture was observed, consistent with compensatory erythropoiesis in this organ (Figures $2 \mathrm{e}$ and $\mathrm{f}$ ). Mice reconstituted with $B c / x^{t / f t}$ bone marrow cells exhibited the expected erythroid subset proportions as well as normal splenic and bone marrow architecture.

$B C L-X L$ is essential for the survival of reticulocytes. Analysis of mitochondrial content revealed that, as expected, $95 \%$ of reticulocytes contained mitochondria and only mature red blood cells were devoid of mitochondria (Figures 3a and b). Having confirmed that reticulocytes and erythroblasts contain the organelle required for the mitochondrial apoptotic pathway, we examined whether the loss of reticulocytes resulting from the absence of BCL-XL was caused by apoptosis. Wild-type reticulocytes were FACS-sorted and treated in vitro with $\mathrm{BH} 3$-mimetic drugs that specifically inhibit BCL-XL (A-1331852) ${ }^{38}$ or BCL-2 (ABT-199; used as a control) (Figure $3 \mathrm{C}$ ). Treatment with $\mathrm{A}-1331852$ resulted in the rapid death of reticulocytes, and this could be substantially reduced during the first $24 \mathrm{~h}$ by blocking caspase activation with QVDOPH. In contrast, treatment with ABT-199 had no effect on reticulocyte viability (Figure $3 \mathrm{c}$ ). Interestingly, the marked dependency on BCL-XL for survival was specific to reticulocytes, as orthochromatic erythroblasts were far less sensitive to $A-1331852$. Furthermore, following $B c / x$ gene deletion a significant increase in the exposure of phosphatidyl-serine (detected by Annexin V staining) - an early apoptotic marker was observed on reticulocytes, but not mature red blood cells, from the bone marrow and spleen (Figure 3d).

BCL-XL was implicated in mitochondria specific-autophagy (mitophagy) owing to the established role of Nip3-like protein$X(N I X)$ in the clearance of mitochondria from maturing red blood cells ${ }^{39}$ and the ability of BCL-XL to interact with the NIX homologue BNIP3. ${ }^{40}$ To investigate the involvement of mitophagy in the loss of reticulocytes caused by BCL-XL deletion, cell viability was measured following treatment of erythroid progenitors with the autophagy inhibitor - 
a

b

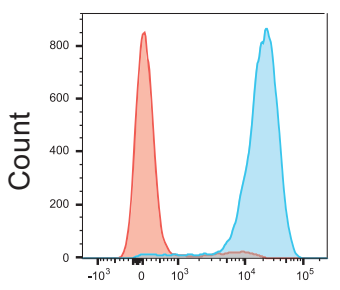

MT Deep Red

reticulocytes

mature tr mature
C

orthochromatic

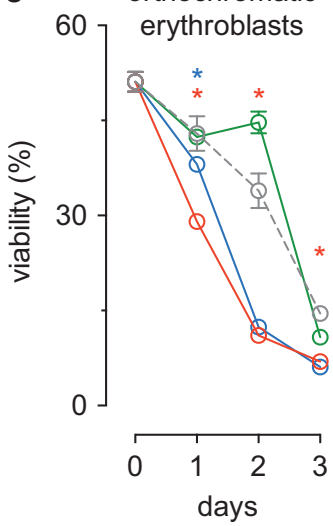

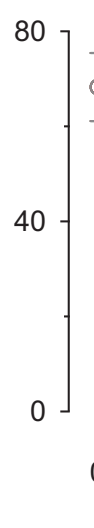

reticulocytes
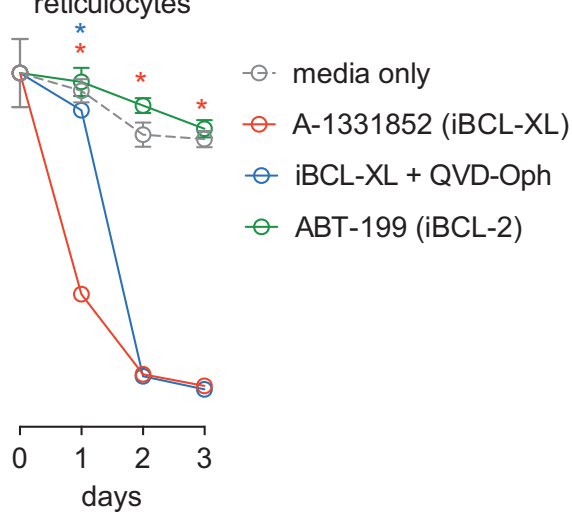
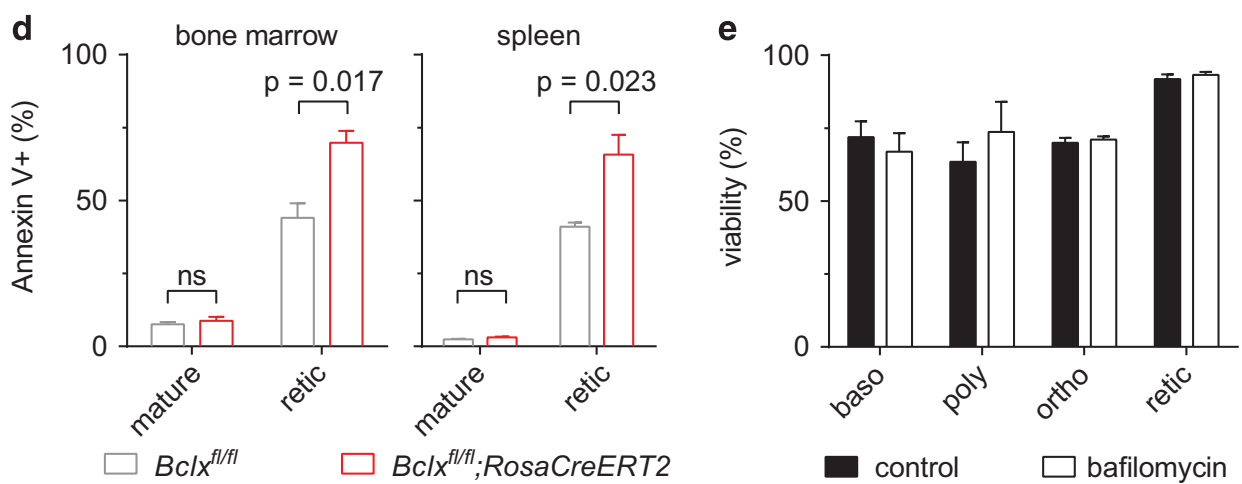

Figure 3 Loss of BCL-XL causes apoptotic death in reticulocytes. (a, b) Wildtype pro-erythroblasts (pro), basophilic erythroblasts (baso), polychromatic erythroblasts (poly), orthochromatic erythroblasts (ortho), reticulocytes (retic) and mature red blood cells (mature) were identified by flow cytometry by their FSC versus CD44 profile and the proportion of cells containing mitochondria was determined by with MitoTracker deep red staining. $n=4$; mean \pm S.E.M.; representative histogram shown. (c) Survival in vitro of FACS-sorted reticulocytes and orthochromatic erythroblasts harvested from wild-type mice. Cells were treated as indicated: A-1331852 (1 $\mu \mathrm{M}, \mathrm{BCL}-\mathrm{XL}), \mathrm{ABT}-199 \mathrm{~g}(1 \mu \mathrm{M}$, iBCL-2), QVD-OPH (25 nM). Cell viability was quantified by forward versus side light scatter profile measured by FACS on days 1-3. Two-way ANOVA analysis with Tukey's multiple comparisons test, comparison between time point means. Stars indicate significant differences: 'red' indicates medium versus iBCL- $\mathrm{XL}$, 'blue' indicates iBCL- $\mathrm{XL}$ versus iBCL-XL plus QVD-OPH. $n=4$, mean \pm S.E.M. shown. (d) Annexin V staining was performed on reticulocytes and mature red blood cells isolated from the bone marrow and spleen of $B c \mid x^{t / f / t} ;$ RosaCreERT2 ${ }^{K /+}$ mice or control $B c l x^{f / f / l}$ mice $24 \mathrm{~h}$ following tamoxifen administration. $n=3$, mean \pm S.E.M. shown. Significant differences indicated with $P$-values as shown, unpaired Students $t$-test, adjusted for multiple testing (Holm-Sidak). (e) Survival in vitro of FACS-sorted basophilic erythroblasts (baso), polychromatic erythroblasts (poly), orthochromatic erythroblasts (ortho), and reticulocytes (retic) from wild-type mice. Cells were treated with bafilomycin (0.1 $\mu \mathrm{M})$ and viability was determined by PI exclusion after $24 \mathrm{~h}$ and compared to untreated cells (control). No significant differences were observed. $n=3$, mean \pm S.E.M. shown

bafilomycin - that prevents fusion between autophagosomes and lysosomes. ${ }^{41}$ Bafilomycin had no impact on erythroid precursor survival (Figure 3e), consistent with the notion that autophagy plays no role in the loss of reticulocytes caused by $\mathrm{BCL}-\mathrm{XL}$ deletion.

Hematopoietic-specific loss of BCL-XL provokes anaemia in adult mice that cannot be alleviated by concomitant loss of BIM or PUMA. We next investigated which proapoptotic BH3-only protein might be critical for reticulocyte apoptosis following loss of BCL-XL. BIM and PUMA are prime candidates because they bind to all pro-survival BCL-2 proteins and are critical for apoptosis induction in many haematopoietic cell types, particularly lymphocytes, ${ }^{20,42-44}$ after exposure to diverse cytotoxic insults. ${ }^{22,45,46}$ To investigate the roles of BIM and PUMA, we reconstituted lethally irradiated mice with bone

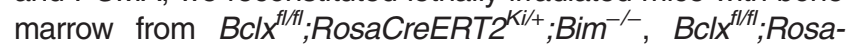
CreERT2 $2^{K /+} ;$ Puma $^{-/-}$or relevant control mice (Figure 4a). Mice expressing GFP in all cells, importantly including erythroid cells, ${ }^{47}$ were used as recipients to distinguish donor-derived haematopoietic cells $\left(\mathrm{GFP}^{-}\right)$from residual recipient-derived cells $\left(\mathrm{GFP}^{+}\right)$(Supplementary Figure 5).

Efficient haematopoietic reconstitution was confirmed 8 weeks post-transplantation with donor-derived $\left(\mathrm{GFP}^{-}\right)$red blood cells present at $>95 \%$ (Figure $4 b$ ). Bclx gene deletion was induced with tamoxifen and after 1 month bone marrow, spleen and peripheral blood cells were analysed. As expected, ${ }^{26}$ tamoxifen-treated $B c / x^{f / f / l} ;$ RosaCreERT2 ${ }^{K / /+}$ reconstituted mice developed severe anaemia evident by low red blood cell numbers and splenomegaly, whereas RosaCreERT2 $2^{K i / 4}$ reconstituted mice presented with normal blood counts and spleen cellularity (Figure 4c). Surprisingly, all

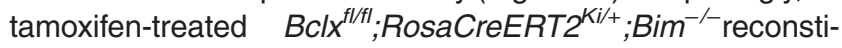
tuted mice presented with splenomegaly and anaemia (Figure 4c). Hence, in contrast to its importance in embryonic erythropoiesis, loss of BIM failed to mitigate the erythropoietic insufficiency caused by BCL-XL deletion in adult mice. Loss of PUMA also provided no protection. Accordingly, no differences in 


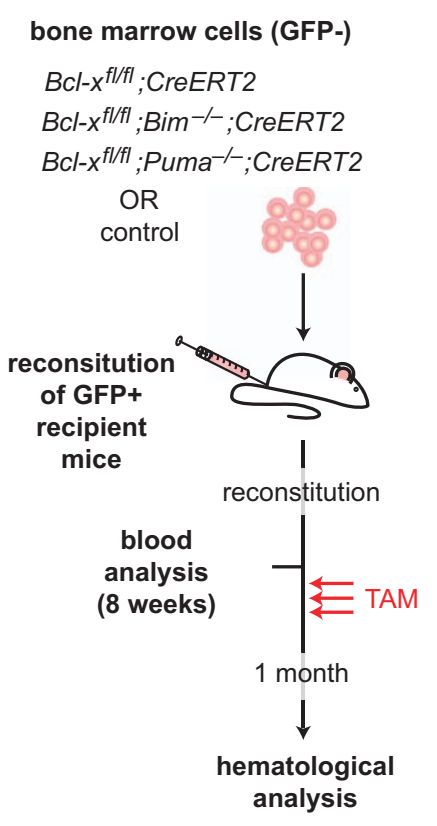

b
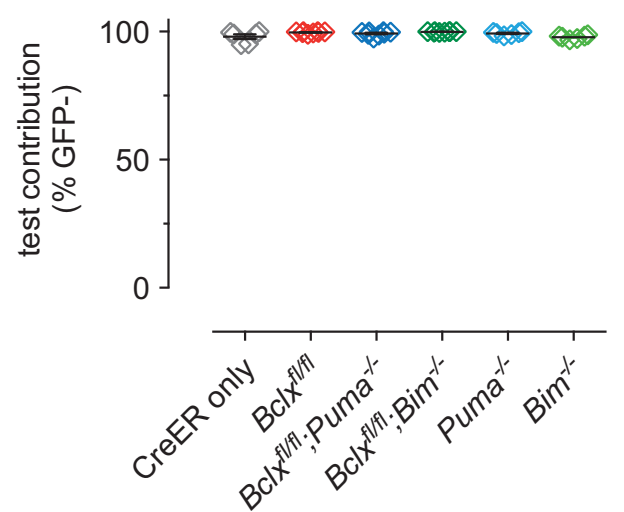
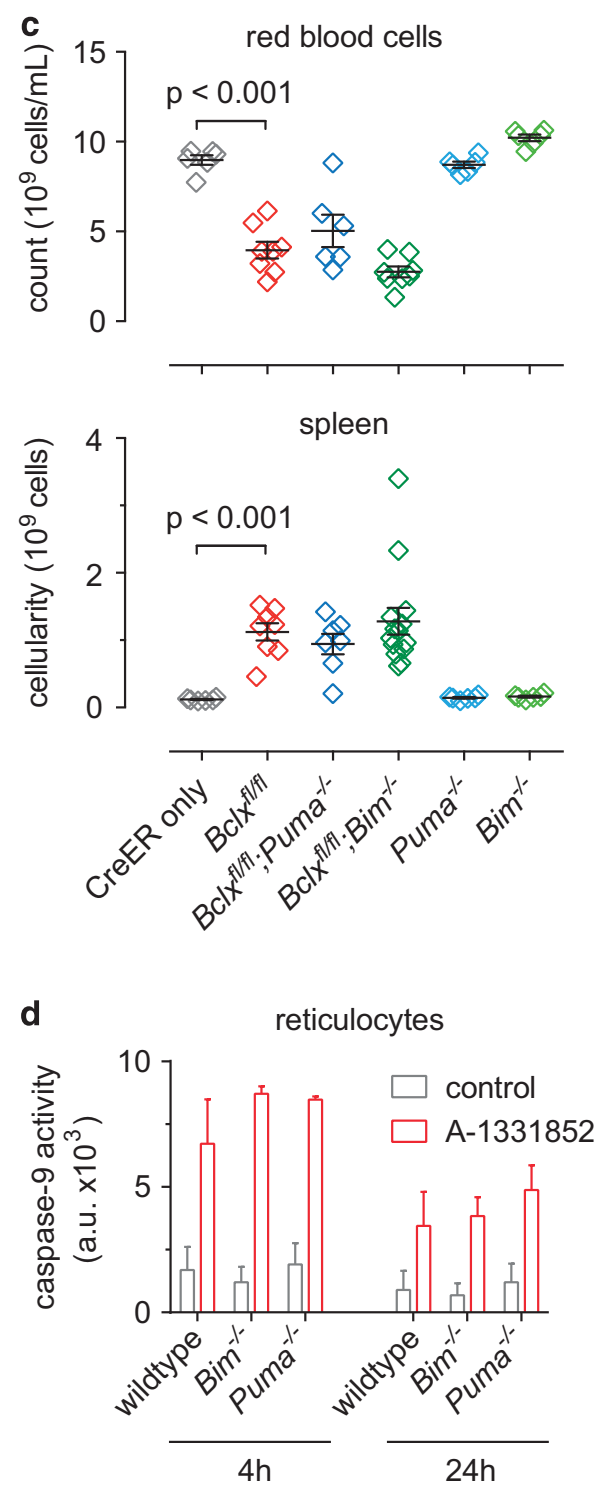

Figure 4 Concomitant loss of BIM or PUMA fails to rescue anaemia caused by acute deletion of $B c l X$. (a) Lethally irradiated GFP' recipient mice were reconstituted with 'test' bone marrow of the indicated genotypes (GFP ${ }^{-}$). At 8 weeks after reconstitution a mandible bleed was taken from each mouse and analysed to confirm successful haematopoietic reconstitution and to establish baseline blood cell counts. Mice were then treated with tamoxifen (TAM, red arrows). Peripheral blood and spleens were collected for analysis 1 month after TAM administration. (b) Test red blood cell contribution at 8 weeks, prior to TAM treatment. $n=6-8(N=3-4$ bone marrow donors); mean \pm S.E.M shown. (c) Red blood cell counts and spleen cellularity after TAM treatment. $n=6-14$ ( $N=3-7$ bone marrow donors); mean \pm S.E.M. (d) In vitro Caspase-9 activity in FACS-sorted reticulocytes, control versus A-1331852-treated $(1 \mu \mathrm{M})$. a.u. denotes arbitrary units. Significant differences as shown, determined by unpaired Student's $t$-test

caspase- 9 activity were observed between reticulocytes from wild-type, $\mathrm{Bim}^{-/}$or Puma ${ }^{-/}$mice following treatment with the BCL-XL-selective inhibitor A-1331852 (Figure 4d).

These findings show that on their own BIM and PUMA are not essential for the loss of reticulocytes and anaemia caused by $\mathrm{BCL}-\mathrm{XL}$ deletion.

PUMA has a minor role in anaemia induced in adult mice by acute BCL-XL loss. To further investigate whether BIM or PUMA may have a minor role in the apoptosis of erythroid cells caused by BCL-XL loss, we adopted a mixed bone marrow reconstitution approach (Figure $4 \mathrm{a}$ ). $\mathrm{GFP}^{+}$mice were reconstituted with a 1:1 mix of bone marrow cells comprising wild-type $\left(\mathrm{GFP}^{+}\right)$'competitor' cells and 'test' cells of the following genotypes: RosaCreERT2 ${ }^{K i /+}$, Bclx ${ }^{f / f l} ;$ RosaCreERT2 ${ }^{K i /+}, \quad B c l x^{f l / f l} ;$ RosaCreERT2 ${ }^{K i /+} ; \mathrm{Bim}^{-/-}$and $B c l x^{f l / f l}$; RosaCreERT2 ${ }^{K i /+} ;$ Puma $^{-/}$(all GFP ${ }^{-}$). As expected, the presence of the wild-type cells prevented the anaemia caused by $\mathrm{BCL}-\mathrm{XL}$ deletion (Figure $5 \mathrm{a}$ ). When the ratio of test to competitor derived cells was determined within the red blood cell compartment, relative to the RosaCreERT2 $2^{K i /+}$ control cells, the $B c / x^{f / f f l} ;$ RosaCreERT2 ${ }^{K i /+}$ cells were profoundly depleted (Figure 5b). This deficit was mildly alleviated by concomitant loss of PUMA, whereas loss of BIM had no impact (Figure $5 b$ ). These results indicate that PUMA has a minor role in the loss of red blood cells resulting from acute BCL-XL loss. 
a

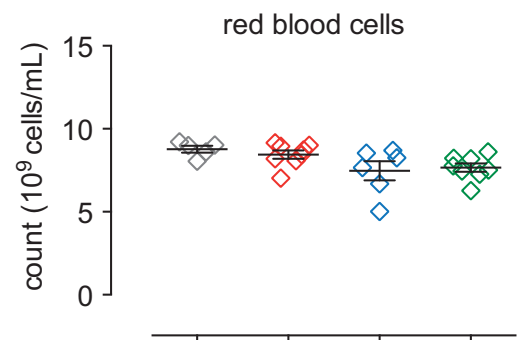

b

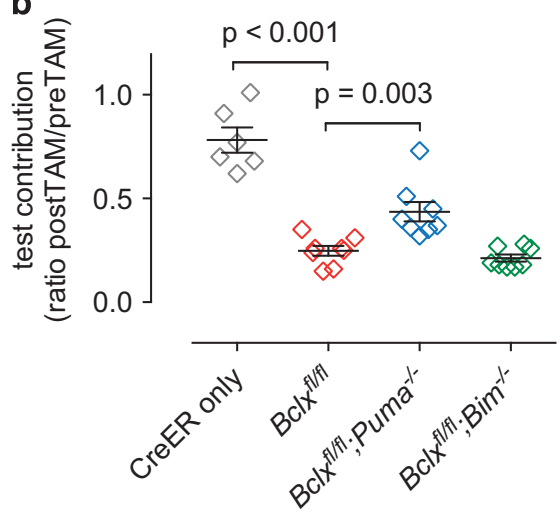

Figure 5 Loss of PUMA partially alleviates impaired erythropoiesis caused by acute deletion of $B c / x$. Lethally irradiated $\mathrm{GFP}^{+}$mice $(2 \times 5.5 \mathrm{~Gy})$ were reconstituted with a 1-to-1 mixture of bone marrow cells from wild-type mice ('competitor'; GFP') and mice of the indicated genotypes ('test'; GFP $^{-}$). Eight weeks after reconstitution blood was taken from reconstituted mice and analysed for test versus competitor contribution. $B c / x$ deletion was then induced by administering tamoxifen. Mice were killed 1 month after tamoxifen administration and peripheral blood was collected for analysis. (a) Overall total red blood cell counts including both $\mathrm{GFP}^{+}$and $\mathrm{GFP}^{-}$ populations. $n=5-8$ ( $N=3-4$ bone marrow donors); mean \pm S.E.M. (b) Ratio of test $\left(\right.$ GFP $\left.^{-}\right)$contribution to red blood cell population pre- and post-tamoxifen treatment. $n=5-8(N=3-4$ bone marrow donors); mean \pm S.E.M. Significant differences as shown, determined by unpaired Student's $t$-test

\section{Discussion}

Throughout life large numbers of red blood cells must be continuously produced in the bone marrow to offset their ongoing loss in the periphery. The high rate of proliferation of progenitors and the marked changes in cellular architecture during differentiation must impose stresses that activate apoptosis signalling. Thus, inhibitors of apoptosis are expected to be critical to sustain survival of differentiating erythroid cells and previous studies identified BCL-XL as the critical factor. Germline deficiency for BCL-XL causes embryonic lethality around E13.5 owing to aberrant death of erythroid and neuronal cells. ${ }^{24}$ Loss of BIM was shown to reduce the excess apoptosis of haematopoietic fetal liver cells in $B c / x^{-/-}$mice (although this did not prevent embryonic lethality). ${ }^{25}$ Studies in vitro concluded that BCL-XL is needed for the survival of late-stage erythroid progenitors. ${ }^{30,48}$ However, the precise stage during erythropoiesis at which $B C L-X L$ is critical for survival was not resolved.

Cell type-specific conditional $B c / x$ gene deletion in adult mice caused severe anaemia, accompanied by splenomegaly and elevated numbers of erythroblasts. This led to the conclusion that $B C L-X L$ is only essential during terminal erythroid maturation. Because of the elevated reticulocyte numbers, the authors of that study attributed the anaemia to excessive haemolysis. ${ }^{26}$ Conversely, a subsequent study using the same model, but a later time point for analysis, reported reduced reticulocyte numbers. The authors of the second study concluded that an erythrocyte production defect was responsible for the anaemia caused by loss of BCL-XL and proposed that mature erythroblasts rather than reticulocytes depend on BCL-XL for survival. ${ }^{31}$

Using genetic and pharmacological approaches coupled with FACS analysis to delineate discrete stages of erythroid differentiation, ${ }^{36}$ we found that $B C L-X L$ is essential for reticulocyte survival but largely dispensable for the survival of orthochromatic erythroblasts and earlier progenitors. This is consistent with reports that the highest levels of BCL-XL are observed during terminal erythrocyte differentiation when haemoglobin production is maximal. ${ }^{8}$ Apoptosis of reticulocytes following acute Bclx gene deletion - documented by appearance of apoptosis-specific markers - results in a compensatory increase in erythroblasts in the bone marrow and the spleen. Our findings clarify previously reported observations. Our use of the RosaCreERT2 allele enabled efficient inducible $B c / x$ gene deletion resulting in anaemia within 1 month, compared with the 3-4 months in the previously described models ${ }^{26}$. This allowed primary effects of BCL-XL loss to be studied in the absence of potentially confounding secondary effects or compensatory processes. Our acute $B c / x$ deletion model closely mimics the scenario of therapeutic BCL-XL inhibition by $\mathrm{BH}$-mimetic drugs and therefore provides insight into their likely impact on patients.

Pro-apoptotic PUMA and BIM were investigated as potential initiators of reticulocyte death caused by loss of BCL-XL. In contrast to the published role for BIM as a critical initiator of erythrocyte precursor death during embryogenesis in $\mathrm{Bcl}$ deficient mice, ${ }^{25}$ we found that concomitant loss of BIM did not alleviate the anaemia caused by acute BCL-XL loss in the adult. Loss of PUMA also provided no rescue. However, experiments using mixed bone marrow reconstituted mice provided evidence that PUMA plays a minor role in the reticulocyte death. Hence, we conclude that other $\mathrm{BH} 3-$ only proteins may be critical (either by themselves or together with PUMA and BIM) for the induction of apoptosis following BCL-XL loss in reticulocytes.

Our findings demonstrate that reticulocytes are the key erythroid precursors that require $\mathrm{BCL}-\mathrm{XL}$ for survival and that acute loss of BCL-XL results in anaemia owing to increased apoptosis of reticulocytes and thus diminished production of mature red blood cells. In mice, mature red blood cells spontaneously lyse after 8-20 days, unless taken up by macrophages for retrieval of iron. Hence, anaemia will ensue upon loss of BCL-XL-dependent reticulocytes, although in mice this can be delayed by compensatory erythropoiesis in the spleen. This accounts for the relatively slow development of anaemia, the low numbers of reticulocytes as well as the elevated numbers of the more primitive erythroid precursors.

In humans, red blood cells survive in the circulation for up to 120 days, significantly longer than mice. The extended survival of red blood cells in humans may provide a therapeutic window for intervention with BCL-XL inhibitors, unless erythropoiesis is already compromised prior to treatment. 
Materials and Methods

Reagents. The BCL-2 inhibitor ABT-199 (ref. 38) was provided by AbbVie and QVD-OPH was purchased from MP Biomedical (\#03OPH10903, Santa Ana, CA, USA). ${ }^{49}$ The BCL-XL inhibitor A-1331852 was prepared according to procedures described in the literature (patent: Wang et al., WO 2013055897). ${ }^{38}$ Bafilomycin was purchased from Sigma Aldrich (\#B1793, St. Louis, MO, USA). Tamoxifen was purchased from Sigma Aldrich.

Mice. All experiments with mice were conducted according to the guidelines of The Walter and Eliza Hall Institute of Medical Research Animal Ethics Committee. Mouse strains utilised in this study have been previously published $\left(B c x^{f / 26} \mathrm{Bim}^{-/ 2}, 20\right.$ Puma $^{-/-}, 42$ UBC-GFP Tg ${ }^{47}$ and RosaCreERT $2^{37}$ ). All mice were either generated on a C57BL/6 background or crossed onto this background for at least 20 generations before commencement of our studies. Tamoxifen administration was performed by ora gavage, $3.6 \mu \mathrm{g} /$ day on 3 consecutive days. Blood transfusion was performed with blood isolated from UBC-GFP Tg mice (GFP'), $250 \mu$ l blood/recipient.

Genotyping was performed as previously reported. ${ }^{22}$ Oligonucleotide sequences for genotyping of the mutant alleles will be provided on request.

Generation of bone marrow chimaeric mice. Haematopoietic reconstitutions were performed as previously described. ${ }^{22}$

Histology and blood film analysis. Sternum and spleen specimens were fixed in $10 \%$ formalin. Paraffin tissue sections were prepared at $7.5 \mu \mathrm{m}$ and stained with haematoxylin and eosin. Images (sternum $\times 20$; spleen $\times 40$ ) were taken with an Olympus BX43 (Olympus, Shinyuku, Japan) using the acquisition program CellSens Standard (Olympus). Slides for analysis of blood cell morphology were prepared by spreading freshly venesected blood by hand onto slides, which were allowed to air-dry. Blood films were fixed in methanol and stained using May-Grunwald and Giemsa stains. Blood film images were acquired using a Nikon Eclipse 90i microscope. Images presented were taken using a $\times 100$ oil objective and inset views for reticulocyte features utilised digital zoom.

Serum analysis. Blood from mice was collected 1 month after tamoxifen treatment via cardiac puncture. Bleeds were left to coagulate at room temperature for $5 \mathrm{~min}$ and centrifuged for $1 \mathrm{~min}$ at $13000 \mathrm{rpm}$ to collect serum. Sera were stored at $-20^{\circ} \mathrm{C}$ and analysed using Architect c1600 (Abbott Diagnostics, Santa Clara, CA, USA).

Flow cytometric analysis. Spleen and bone marrow cells were harvested and single-cell suspensions prepared. Cells were counted using the CasyCell Counter (Schaefe System GmbH, Neunkirchen, Germany). Retro-orbital bleeds were collected into EDTA for differential cell counts using an ADVIA 2120 analyser (Siemens Healthcare PTY, Ltd, Bayswater, VIC, Australia). The analysis of red blood cells and leukocytes from either wild-type or test bone marrow in the haematopoietic reconstituted mice was determined by flow cytometric analysis of GFP and staining for haematopoietic subset-specific surface markers (CD44 [IM781], TER119 [TER119], B220 [RA3-6B2] or CD19 [ID3], GR-1 [RB6-8C5], MAC-1 [MI/70], CD45.2 [5.450.15.2], CD3 [KT3-1.1]). Antibodies were conjugated to FITC, R-PE or APC. For erythroid precursor analysis, mature cells were gated out prior to CD44 versus FSC analysis using a 'DUMP' channel with the following surface markers: B220 or CD19, GR-1, MAC-1, CD45.2, CD3. GFP ${ }^{+}$(wild-type bone marrow derived) and GFP- (test bone marrow derived) cells were used to determine relative contributions in the haematopoietic reconstituted mice. Samples were analysed in a LSR-II flow cytometer (BD Biosciences, San Jose, CA, USA). Dead cells were excluded using forward and side light scatter plus propidium iodide exclusion (Sigma Aldrich).

For stem and progenitor cell analysis, cells were stained with Alexa Fluor 700 conjugated antibodies against the following surface markers: CD45R (B220), CD19, GR-1, Ly6G, F4/80, TER119, NK1.1, CD2, CD4, and CD8 and CD117 PerCPCy5.5 (c-KIT, clone 2B8 BD Biosciences), SCA-1 Alexa Fluor 594, CD135 PE (FLT3/FLK2 clone A2F10 Biolegend, San Diego, CA, USA), CD34 Alexa Fluor 647 (clone RAM34 BD Biosciences) $\mathrm{CD} 16 / 32$ biotin (Fc $\gamma$ receptor II/III clone 24G2 BD Biosciences) and Streptavidin BV650 (BD Biosciences) and analysed in a Fortessa I flow cytometer (BD Bioscience). Dead cells were excluded using forward and side light scatter and Fluoro-Gold (Fluorochrome LLC, Denver, CO, USA) exclusion. Mature cells were gated out using the Alexa Fluor 700 (Lineage) 'DUMP' channel.

Mitochondrial content analysis. Cells were incubated with $500 \mathrm{nM}$ MitoTracker Deep Red for $20 \mathrm{~min}$ at $37^{\circ} \mathrm{C}$ (Thermo Fisher, \#M22426). Cells were then stained for the appropriate lineage markers, TER119 and CD44 to delineate specific erythroid cell populations by flow cytometry.

Caspase-9 activity assay. $5 \times 10^{4}$ bone marrow cells were cultured for 4 or $24 \mathrm{~h}$ with or without the BCL-XL inhibitor A-1331852 in a 96-well plate in $100 \mu \mathrm{l}$ of medium. Plates were allowed to equilibrate at room temperature prior to adding $100 \mu$ l Caspase-9 Glo reagent (Promega, Madison, WI, USA). Assays were analysed after 30 min incubation according to the manufacturer's instructions.

Clonogenic assays. CFU-e were enumerated by culturing single-cell suspensions of bone marrow $(25000)$ or spleen $(50000)$ in $1 \mathrm{ml}$ of MethoCult 3234 medium (Stem Cell Technologies, Vancouver, BC, Canada) supplemented with $2 \mathrm{U} / \mathrm{ml}$ erythropoietin (EPO, Janssen-Cilag Ltd, Buckinghamshire, UK) with incubation in $5 \% \mathrm{CO}_{2}$ in air for 2-3 days. The numbers of myeloid colonyforming cells in single-cell suspensions of bone marrow (25 000) or spleen (50 000) were assessed in $1 \mathrm{ml}$ cultures of $0.3 \%$ agar in Dulbecco/s modified Eagles medium containing 20\% newborn calf serum, stem cell factor (SCF, $100 \mathrm{ng} / \mathrm{ml}$, WEHI), EPO (2 U/ml, Janssen-Cilag Ltd) and interleukin-3 (IL-3, $10 \mathrm{ng} / \mathrm{ml})$. Cultures were incubated at $37^{\circ} \mathrm{C}$ for 7 days in $10 \% \mathrm{CO}_{2}$ in air. Cultures were fixed, dried onto glass slides and stained for acetylcholinesterase followed by Luxol fast blue and haematoxylin to determine the numbers and type of colonies.

Cell survival assays in vitro. Bone marrow cells were collected and stained with antibodies against TER119 (TER119) and CD44 (IM781) and a lineage marker cocktail (CD3 [KT3], MAC-1 [MI/70], GR-1 [RB6-8C5], B220 [RA3-6B2], CD45.2 [5.450.15.2]). Lineage ${ }^{-}$TER119 ${ }^{+}$cells were sorted using the Aria cell sorter (BD) and further subdivided based on CD44 staining and forward light scatter. Reticulocytes and orthochromatic reticulocytes were resuspended in Dulbecco's medium (Invitrogen, Life Technologies, Carlsbad, CA, USA) supplemented with 10\% FCS (Bovogen, East Keilor, VIC, Australia), $100 \mathrm{mM}$ asparagine (Sigma Aldrich) and $50 \mathrm{mM}$ 2-mercapoethanol (Sigma Aldrich) without addition of cytokines. Cells were treated either with DMSO (control; $1 \times 10^{-4} \%$; Sigma Aldrich), $1 \mu \mathrm{M}$ of A-1331852 (provided by AbbVie, North Chicago, IL, USA), $1 \mu \mathrm{M}$ A-1331852+25 nM QVD-OPH (MP Biomedicals) or $1 \mu \mathrm{M}$ ABT-199. Cells were collected after 0, 24, 48 and $72 \mathrm{~h}$ and viability was determined by FACS analysis using forward by side light scatter profile to gate for live cells or Annexin V staining.

Statistical analysis. Cellularity, weight, blood parameters, flow cytometric results and in vitro cell survival were plotted and analysed with GraphPad Prism (GraphPad Software Inc, La Jolla, CA, USA). Statistical comparisons were conducted in a pair-wise manner using unpaired two-tailed Student's $t$-test assuming equal variance. For Figure $2 \mathrm{c}$ we utilised the Sidak-Bonferroni method for multiple comparisons correction. For Figure 4 we performed two-way ANOVA analysis with Tukey's multiple comparisons test. Stars indicate significant values. Error bars are presented as standard error of mean $( \pm$ S.E.M.).

\section{Conflict of Interest}

The authors declare no conflict of interest.

Acknowledgements. We thank Drs P Bouillet, JM Adams, L Hennighausen, and A Villunger for gifts of mice; C Gatt, S O'Connor, J Mansheim, K McKenzie and G Siciliano for expert animal care; B Helbert for genotyping; J Corbin and J McManus for automated blood analysis. This work was supported by grants and fellowships from the Cancer Council of Victoria (SG, AD 'Sydney Parker Smith Postdoctoral Fellowship'), Leukaemia Foundation Australia (SG, BJA), the Lady Tata Memorial Trust (SG), Cure Brain Cancer Australia (AS), the National Health and Medical Research Council (Programme Grants \#1016701 and 1016647, NHMRC Australia Fellowship 1020363 to AS and Research Fellowship 1058344 to WSA), the Leukemia and Lymphoma Society (SOCR Grant \#7001-03 to AS), The Australian Cancer Research Fund (GL, JG), Melbourne International Research and the Melbourne International Fee Remission Scholarship (University of Melbourne, SG) and Cancer Therapeutics CRC Top-up Scholarship (SG, AD). The estate of Anthony (Toni) Redstone OAM, University of Melbourne International Research and International Fee Remission Scholarships (SG), Australian Postgraduate Award (ARDD, JPB), and the operational infrastructure grants through the Australian Government IRIISS and the Victorian State Government OIS. 


\section{Author contributions}

This study was conceived by $A D$ and $S G$ with guidance from $A S$. Experiments were designed, conducted and analysed by AD, SG, WA, BJA, JB, CH, LR and AS. A-1331852 was prepared by JG and GL. Manuscript was prepared by AD, SG, BJA, WA and AS with assistance from the other authors.

1. de Benoist B, McLean E, Egli I, Cogswell M. Worldwide prevalence of anaemia 1993-2005L WHO global database on anaemia. World Health Organisation: Geneva, Switzerland, 2008

2. Wilson WH, O'Connor OA, Czuczman MS, Lacasce AS, Gerecitano JF, Leonard JP et al. Navitoclax, a targeted high-affinity inhibitor of BCL-2, in lymphoid malignancies: a phase 1 dose-escalation study of safety, pharmacokinetics, pharmacodynamics, and antitumour activity. Lancet Oncol 2010; 11: 1149-1159.

3. Roberts AW, Davids MS, Pagel JM, Kahl BS, Puvvada SD, Gerecitano JF et al. Targeting BCL2 with venetoclax in relapsed chronic lymphocytic leukemia. N Engl J Med 2016; 374: 311-322.

4. Koury MJ. Tracking erythroid progenitor cells in times of need and times of plenty. Exp Hematol 2016; 44: 653-663.

5. Barminko J, Reinholt B, Baron MH. Development and differentiation of the erythroid lineage in mammals. Dev Comp Immunol 2016; 58: 18-29.

6. Magnani M, Rossi L, Stocchi V, Cucchiarini L, Piacentini G, Fornaini G. Effect of age on some properties of mice erythrocytes. Mech Ageing Dev 1988; 42: 37-47.

7. Dholakia U, Bandyopadhyay S, Hod EA, Prestia KA. Determination of RBC Survival in C57BL/6 and C57BL/6-Tg(UBC-GFP) Mice. Comp Med 2015; 65: 196-201.

8. Gregoli PA, Bondurant MC. The roles of $\mathrm{BCl}-\mathrm{x}_{\mathrm{L}}$ and apopain in the control of erythropoiesis by erythropoietin. Blood 1997; 90: 630-640.

9. Rund D, Rachmilewitz E. Beta-thalassemia. N Engl J Med 2005; 353: 1135-1146.

10. Weiss G, Goodnough LT. Anemia of chronic disease. N Engl J Med 2005; 352: 1011-1023.

11. Horos R, von Lindern M. Molecular mechanisms of pathology and treatment in Diamond Blackfan Anaemia. Br J Haematol 2012; 159: 514-527.

12. Miyashita T, Krajewski S, Krajewska M, Wang HG, Lin HK, Liebermann DA et al. Tumor suppressor $\mathrm{p} 53$ is a regulator of bcl-2 and bax gene expression in vitro and in vivo. Oncogene 1994; 9: 1799-1805.

13. Nakano K, Vousden KH. PUMA, a novel proapoptotic gene, is induced by p53. Mol Cell 2001; 7: 683-694.

14. Yu J, Zhang L, Hwang PM, Kinzler KW, Vogelstein B. PUMA induces the rapid apoptosis of colorectal cancer cells. Mol Cell 2001; 7: 673-682.

15. Oda E, Ohki R, Murasawa H, Nemoto J, Shibue T, Yamashita T et al. Noxa, a BH3-only member of the bcl-2 family and candidate mediator of p53-induced apoptosis. Science 2000; 288: $1053-1058$

16. Delbridge AR, Strasser A. The BCL-2 protein family, BH3-mimetics and cancer therapy. Cell Death Differ 2015; 22: 1071-1080.

17. Czabotar PE, Lessene G, Strasser A, Adams JM. Control of apoptosis by the BCL-2 protein family: implications for physiology and therapy. Nat Rev Mol Cell Biol 2014; 15: 49-63.

18. O'Neill KL, Huang K, Zhang J, Chen Y, Luo X. Inactivation of prosurvival Bcl-2 proteins activates Bax/Bak through the outer mitochondrial membrane. Genes Dev 2016; 30: 973-988.

19. Chipuk JE, Green DR. How do BCL-2 proteins induce mitochondrial outer membrane permeabilization? Trends Cell Biol 2008; 18: 157-164.

20. Bouillet P, Metcalf D, Huang DCS, Tarlinton DM, Kay TWH, Köntgen F et al. Proapoptotic $\mathrm{Bcl}-2$ relative Bim required for certain apoptotic responses, leukocyte homeostasis, and to preclude autoimmunity. Science 1999; 286: 1735-1738

21. Mason KD, Lin A, Robb L, Josefsson EC, Henley KJ, Gray DH et al. Proapoptotic Bak and Bax guard against fatal systemic and organ-specific autoimmune disease. Proc Natl Acad Sci USA 2013; 110: 2599-2604.

22. Delbridge AR, Opferman JT, Grabow S, Strasser A. Antagonism between MCL-1 and PUMA governs stem/progenitor cell survival during hematopoietic recovery from stress. Blood 2015; 125: 3273-3280.

23. Opferman J, Iwasaki H, Ong CC, Suh H, Mizuno S, Akashi K et al. Obligate role of antiapoptotic MCL-1 in the survival of hematopoietic stem cells. Science 2005; 307: 1101-1104.

24. Motoyama N, Wang FP, Roth KA, Sawa H, Nakayama K, Nakayama K et al. Massive cell death of immature hematopoietic cells and neurons in Bcl-x deficient mice. Science 1995; 267: $1506-1510$.

25. Akhtar RS, Klocke BJ, Strasser A, Roth KA. Loss of BH3-only protein Bim inhibits apoptosis of hemopoietic cells in the fetal liver and male germ cells but not neuronal cells in bcl-x-deficient mice. J Histochem Cytochem 2008; 56: 921-927.

26. Wagner KU, Claudio E, Rucker EB 3rd, Riedlinger G, Broussard C, Schwartzberg PL et al. Conditional deletion of the $\mathrm{Bcl}-\mathrm{x}$ gene from erythroid cells results in hemolytic anemia and profound splenomegaly. Development 2000; 127: 4949-4958.

27. Oltersdorf T, Elmore SW, Shoemaker AR, Armstrong RC, Augeri DJ, Belli BA et al. An inhibitor of Bcl-2 family proteins induces regression of solid tumours. Nature 2005; 435: 677-681.

28. Tse C, Shoemaker AR, Adickes J, Anderson MG, Chen J, Jin S et al. ABT-263: a potent and orally bioavailable Bcl-2 family inhibitor. Cancer Res 2008; 68: 3421-3428.
29. Souers AJ, Leverson JD, Boghaert ER, Ackler SL, Catron ND, Chen J et al. ABT-199, a potent and selective BCL-2 inhibitor, achieves antitumor activity while sparing platelets. Nat Med 2013; 19: 202-208.

30. Motoyama N, Kimura T, Takahashi T, Watanabe T, Nakano T. bcl-x prevents apoptotic cell death of both primitive and definitive erythrocytes at the end of maturation. J Exp Med 1999; 189: $1691-1698$.

31. Rhodes MM, Kopsombut P, Bondurant MC, Price JO, Koury MJ. Bcl- $\mathrm{x}(\mathrm{L})$ prevents apoptosis of late-stage erythroblasts but does not mediate the antiapoptotic effect of erythropoietin. Blood 2005; 106: 1857-1863

32. Mason KD, Carpinelli MR, Fletcher JI, Collinge JE, Hilton AA, Ellis S et al. Programmed anuclear cell death delimits platelet life span. Cell 2007; 128: 1173-1186.

33. Zhang H, Nimmer PM, Tahir SK, Chen J, Fryer RM, Hahn KR et al. Bcl-2 family proteins are essential for platelet survival. Cell Death Differ 2007; 14: 943-951.

34. Delbridge AR, Chappaz S, Ritchie ME, Kile BT, Strasser A, Grabow S. Loss of PUMA (BBC3) does not prevent thrombocytopenia caused by the loss of BCL-XL (BCL2L1). Br J Haematol 2016; 174: 962-969.

35. Kodama T, Hikita H, Kawaguchi T, Shigekawa M, Shimizu S, Hayashi Y et al. Mcl-1 and Bcl$\mathrm{XL}$ regulate Bak/Bax-dependent apoptosis of the megakaryocytic lineage at multistages. Cell Death Differ 2012; 19: 1856-1869.

36. Liu J, Zhang J, Ginzburg Y, Li H, Xue F, De Franceschi L et al. Quantitative analysis of murine terminal erythroid differentiation in vivo: novel method to study normal and disordered erythropoiesis. Blood 2013; 121: e43-e49.

37. Seibler J, Zevnik B, Kuter-Luks B, Andreas S, Kern H, Hennek T et al. Rapid generation of inducible mouse mutants. Nucleic Acids Res 2003; 31: e12.

38. Leverson JD, Phillips DC, Mitten MJ, BoghaertER, Diaz D, Tahir SK et al. Exploiting selective $\mathrm{BCL}-2$ family inhibitors to dissect cell survival dependencies and define improved strategies for cancer therapy. Sci Transl Med 2015; 7: 279ra240.

39. Schweers RL, Zhang J, Randall MS, Loyd MR, Li W, Dorsey FC et al. NIX is required for programmed mitochondrial clearance during reticulocyte maturation. Proc Natl Acad Sci USA 2007; 104: 19500-19505.

40. Ray R, Chen G, Vande Velde C, Cizeau J, Park JH, Reed JC et al. BNIP3 heterodimerizes with $\mathrm{Bcl}-2 / \mathrm{Bcl}-\mathrm{X}(\mathrm{L})$ and induces cell death independent of a $\mathrm{Bcl}-2$ homology $3(\mathrm{BH} 3)$ domain at both mitochondrial and nonmitochondrial sites. J Biol Chem 2000; 275: 1439-1448.

41. Yamamoto A, Tagawa Y, Yoshimori T, Moriyama Y, Masaki R, Tashiro Y. Bafilomycin A1 prevents maturation of autophagic vacuoles by inhibiting fusion between autophagosomes and lysosomes in rat hepatoma cell line, H-4-II-E cells. Cell Struct Funct 1998; 23: 33-42.

42. Villunger A, Michalak EM, Coultas L, Mullauer F, Bock G, Ausserlechner MJ et al. p53- and drug-induced apoptotic responses mediated by $\mathrm{BH}$-only proteins puma and noxa. Science 2003; 302: 1036-1038.

43. Jeffers JR, Parganas E, Lee Y, Yang C, Wang J, Brennan J et al. Puma is an essential mediato of p53-dependent and -independent apoptotic pathways. Cancer Cell 2003; 4: 321-328.

44. Erlacher M, Michalak EM, Kelly PN, Labi V, Niederegger H, Coultas L et al. BH3-only proteins Puma and Bim are rate-limiting for \{gamma\} -radiation and glucocorticoid-induced apoptosis of lymphoid cells in vivo. Blood 2005; 106: 4131-4138.

45. Zhang CZ, Zhang JX, Zhang AL, Shi ZD, Han L, Jia ZF et al. MiR-221 and miR-222 target PUMA to induce cell survival in glioblastoma. Mol Cancer 2010; 9: 229.

46. Yu H, Shen $\mathrm{H}$, Yuan $\mathrm{Y}$, Xufeng R, Hu X, Garrison SP et al. Deletion of Puma protects hematopoietic stem cells and confers long-term survival in response to high-dose \{gamma\}irradiation. Blood 2010; 115: 3472-3480.

47. Schaefer BC, Schaefer ML, Kappler JW, Marrack P, Kedl RM. Observation of antigendependent CD8+ T-cell/ dendritic cell interactions in vivo. Cell Immunol 2001; 214: 110-122.

48. Gregory T, Yu C, Ma A, Orkin SH, Blobel GA, Weiss MJ. GATA-1 and erythropoietin cooperate to promote erythroid cell survival by regulating bcl- $\mathrm{X}_{\mathrm{L}}$ expression. Blood 1999; 94: 87-96.

49. Caserta T, Smith A, Gultice A, Reedy M, Brown T. Q-VD-OPh, a broad spectrum caspase inhibitor with potent antiapoptotic properties. Apoptosis 2003; 8: 345-352.

Cell Death and Disease is an open-access journal published by Nature Publishing Group. This work is licensed under a Creative Commons Attribution 4.0 International License. The images or other third party material in this article are included in the article's Creative Commons license, unless indicated otherwise in the credit line; if the material is not included under the Creative Commons license, users will need to obtain permission from the license holder to reproduce the material. To view a copy of this license, visit http://creativecommons.org/licenses/by/4.0/

(C) The Author(s) 2017 